CUBO A Mathematical Journal

Vol.14, No 03, (71-83). October 2012

\title{
Fractional Voronovskaya type asymptotic expansions for quasi-interpolation neural network operators
}

\author{
George A. Anastassiou \\ Department of Mathematical Sciences, \\ University of Memphis, \\ Memphis, TN 38152, U.S.A. \\ email: ganastss@memphis.edu
}

\begin{abstract}
Here we study further the quasi-interpolation of sigmoidal and hyperbolic tangent types neural network operators of one hidden layer. Based on fractional calculus theory we derive fractional Voronovskaya type asymptotic expansions for the error of approximation of these operators to the unit operator.
\end{abstract}

\section{RESUMEN}

Estudiamos la cuasi-interpolación de los operadores de redes neuronales de tipo tangencial hiperbólico y sigmoidal de una capa oculta. Basados en la Teoría del Cálculo Fraccional, obtenemos expansiones asintóticas del tipo Voronovskaya para el error en la aproximación de estos operadores hacia el operador unitario.

Keywords and Phrases: Neural Network Fractional Approximation, Voro- novskaya Asymptotic Expansion, fractional derivative.

2010 AMS Mathematics Subject Classification: 26A33, 41A25, 41A36, 41A60. 


\section{Background}

We need

Definition 1. Let $v>0, n=\lceil v\rceil\left(\lceil\cdot\rceil\right.$ is the ceiling of the number), $f \in A C^{n}([a, b])$ (space of functions $f$ with $f^{(n-1)} \in A C([a, b])$, absolutely continuous functions). We call left Caputo fractional derivative (see [13], pp. 49-52) the function

$$
D_{* a}^{v} f(x)=\frac{1}{\Gamma(n-v)} \int_{a}^{x}(x-t)^{n-v-1} f^{(n)}(t) d t
$$

$\forall x \in[a, b]$, where $\Gamma$ is the gamma function $\Gamma(v)=\int_{0}^{\infty} e^{-t} t^{v-1} d t, v>0$. Notice $D_{* a}^{v} f \in L_{1}([a, b])$ and $D_{* a}^{v} f$ exists a.e.on $[a, b]$.

We set $D_{* a}^{0} f(x)=f(x), \forall x \in[a, b]$.

Definition 2. (see also [3], 14], [15]). Let $f \in A C^{m}([a, b]), m=\lceil\alpha\rceil, \alpha>0$. The right Caputo fractional derivative of order $\alpha>0$ is given by

$$
D_{b-}^{\alpha} f(x)=\frac{(-1)^{m}}{\Gamma(m-\alpha)} \int_{x}^{b}(\zeta-x)^{m-\alpha-1} f^{(m)}(\zeta) d \zeta
$$

$\forall x \in[a, b]$. We set $D_{b-}^{0} f(x)=f(x)$. Notice $D_{b-}^{\alpha} f \in L_{1}([a, b])$ and $D_{b-}^{\alpha} f$ exists a.e.on $[a, b]$.

Convention 3. We assume that

$$
D_{* x_{0}}^{\alpha} f(x)=0, \text { for } x<x_{0}
$$

and

$$
D_{x_{0}-}^{\alpha} f(x)=0, \text { for } x>x_{0}
$$

for all $x, x_{0} \in[a, b]$.

We mention

Proposition 4. (by [5]) Let $f \in \mathrm{C}^{n}([\mathrm{a}, \mathrm{b}]), \mathrm{n}=\lceil\boldsymbol{v}\rceil, v>0$. Then $\mathrm{D}_{* \mathrm{a}}^{v} \mathrm{f}(\mathrm{x})$ is continuous in $x \in[a, b]$.

Also we have

Proposition 5. (by [5]) Let $f \in C^{m}([a, b]), m=\lceil\alpha\rceil, \alpha>0$. Then $D_{b-}^{\alpha} f(x)$ is continuous in $x \in[a, b]$.

Theorem 6. ([5]) Let $f \in \mathrm{C}^{\mathrm{m}}([\mathrm{a}, \mathrm{b}]), \mathrm{m}=\lceil\alpha\rceil, \alpha>0, x, x_{0} \in[a, b]$. Then $\mathrm{D}_{* \mathrm{x}_{0}}^{\alpha} f(x), \mathrm{D}_{\mathrm{x}_{0}-}^{\alpha} f(x)$ are jointly continuous functions in $\left(x, x_{0}\right)$ from $[a, b]^{2}$ into $\mathbb{R}$.

We mention the left Caputo fractional Taylor formula with integral remainder.

Theorem 7. ([13], p. 54) Let $f \in A C^{m}([a, b]),[a, b] \subset \mathbb{R}, m=\lceil\alpha\rceil, \alpha>0$. Then

$$
f(x)=\sum_{k=0}^{m-1} \frac{f^{(k)}\left(x_{0}\right)}{k !}\left(x-x_{0}\right)^{k}+\frac{1}{\Gamma(\alpha)} \int_{x_{0}}^{x}(x-J)^{\alpha-1} D_{* x_{0}}^{\alpha} f(J) d J,
$$

$\forall x \geq x_{0} ; x, x_{0} \in[a, b]$. 
Also we mention the right Caputo fractional Taylor formula.

Theorem 8. ([3]) Let $f \in A C^{m}([a, b]),[a, b] \subset \mathbb{R}, m=\lceil\alpha\rceil, \alpha>0$. Then

$$
f(x)=\sum_{j=0}^{m-1} \frac{f^{(k)}\left(x_{0}\right)}{k !}\left(x-x_{0}\right)^{k}+\frac{1}{\Gamma(\alpha)} \int_{x}^{x_{0}}(J-x)^{\alpha-1} D_{x_{0}-}^{\alpha} f(J) d J,
$$

$\forall x \leq x_{0} ; x, x_{0} \in[a, b]$

For more on fractional calculus related to this work see [2], 4] and [7].

We consider here the sigmoidal function of logarithmic type

$$
s(x)=\frac{1}{1+e^{-x}}, \quad x \in \mathbb{R} .
$$

It has the properties $\lim _{x \rightarrow+\infty} s(x)=1$ and $\lim _{x \rightarrow-\infty} s(x)=0$.

This function plays the role of an activation function in the hidden layer of neural networks.

As in [12, we consider

$$
\Phi(x):=\frac{1}{2}(s(x+1)-s(x-1)), \quad x \in \mathbb{R} .
$$

We notice the following properties:

i) $\Phi(x)>0, \forall x \in \mathbb{R}$,

ii) $\sum_{k=-\infty}^{\infty} \Phi(x-k)=1, \quad \forall x \in \mathbb{R}$,

iii) $\sum_{\mathrm{k}=-\infty}^{\infty} \Phi(\mathrm{nx}-\mathrm{k})=1, \forall x \in \mathbb{R} ; \mathrm{n} \in \mathbb{N}$,

iv) $\int_{-\infty}^{\infty} \Phi(x) d x=1$,

v) $\Phi$ is a density function,

vi) $\Phi$ is even: $\Phi(-x)=\Phi(x), x \geq 0$.

We see that $([12])$

$$
\begin{aligned}
\Phi(x)= & \left(\frac{e^{2}-1}{2 e}\right) \frac{e^{-x}}{\left(1+e^{-x-1}\right)\left(1+e^{-x+1}\right)}=8 \\
& \left(\frac{e^{2}-1}{2 e^{2}}\right) \frac{1}{\left(1+e^{x-1}\right)\left(1+e^{-x-1}\right)} .
\end{aligned}
$$

vii) By [12 $\Phi$ is decreasing on $\mathbb{R}_{+}$, and increasing on $\mathbb{R}_{-}$. 
viii) By [11] for $n \in \mathbb{N}, 0<\beta<1$, we get

$$
\left\{\begin{array}{l}
\sum_{\substack{k=-\infty \\
:|n x-k|>n^{1-\beta}}}^{\infty} \Phi(n x-k)<\left(\frac{e^{2}-1}{2}\right) e^{-n^{(1-\beta)}}=3.1992 e^{-n^{(1-\beta)}} .
\end{array}\right.
$$

Denote by $\lfloor\cdot\rfloor$ the integral part of a number. Consider $x \in[a, b] \subset \mathbb{R}$ and $n \in \mathbb{N}$ such that $\lceil\mathrm{na}\rceil \leq\lfloor n b\rfloor$.

ix) By [1] it holds

$$
\frac{1}{\sum_{k=\lceil n a\rceil}^{\lfloor n b\rfloor} \Phi(n x-k)}<\frac{1}{\Phi(1)}=5.250312578, \forall x \in[a, b] .
$$

x) By [11] it holds $\lim _{n \rightarrow \infty} \sum_{k=\lceil n a\rceil}^{\lfloor n b\rfloor} \Phi(n x-k) \neq 1$, for at least some $x \in[a, b]$.

Let $f \in C([a, b])$ and $n \in \mathbb{N}$ such that $\lceil n a\rceil \leq\lfloor n b\rfloor$.

We study further (see also [11]) the quasi-interpolation positive linear neural network operator

$$
G_{n}(f, x):=\frac{\sum_{k=\lceil n a\rceil}^{\lfloor n b\rfloor} f\left(\frac{k}{n}\right) \Phi(n x-k)}{\sum_{k=\lceil n a\rceil}^{\lfloor n b\rfloor} \Phi(n x-k)}, \quad x \in[a, b] .
$$

For large enough $n$ we always obtain $\lceil n a\rceil \leq\lfloor n b\rfloor$. Also $a \leq \frac{k}{n} \leq b$, iff $\lceil n a\rceil \leq k \leq\lfloor n b\rfloor$.

We also consider here the hyperbolic tangent function $\tanh x, x \in \mathbb{R}$ :

$$
\tanh x:=\frac{e^{x}-e^{-x}}{e^{x}+e^{-x}}=\frac{e^{2 x}-1}{e^{2 x}+1} .
$$

It has the properties $\tanh 0=0,-1<\tanh x<1, \forall x \in \mathbb{R}$, and $\tanh (-x)=-\tanh x$. Furthermore $\tanh x \rightarrow 1$ as $x \rightarrow \infty$, and $\tanh x \rightarrow-1$, as $x \rightarrow-\infty$, and it is strictly increasing on $\mathbb{R}$. Furthermore it holds $\frac{\mathrm{d}}{\mathrm{dx}} \tanh x=\frac{1}{\cosh ^{2} x}>0$.

This function plays also the role of an activation function in the hidden layer of neural networks.

We further consider

$$
\Psi(x):=\frac{1}{4}(\tanh (x+1)-\tanh (x-1))>0, \quad \forall x \in \mathbb{R} .
$$

We easily see that $\Psi(-x)=\Psi(x)$, that is $\Psi$ is even on $\mathbb{R}$. Obviously $\Psi$ is differentiable, thus continuous.

Here we follow [8]

Proposition 9. $\Psi(x)$ for $x \geq 0$ is strictly decreasing. 
Obviously $\Psi(x)$ is strictly increasing for $x \leq 0$. Also it holds $\lim _{x \rightarrow-\infty} \Psi(x)=0=\lim _{x \rightarrow \infty} \Psi(x)$.

Infact $\Psi$ has the bell shape with horizontal asymptote the $x$-axis. So the maximum of $\Psi$ is at zero, $\Psi(0)=0.3809297$.

Theorem 10. We have that $\sum_{i=-\infty}^{\infty} \Psi(x-i)=1, \forall x \in \mathbb{R}$.

Thus

$$
\sum_{i=-\infty}^{\infty} \Psi(n x-i)=1, \quad \forall n \in \mathbb{N}, \forall x \in \mathbb{R} .
$$

Furthermore we get:

Since $\Psi$ is even it holds $\sum_{i=-\infty}^{\infty} \Psi(i-x)=1, \forall x \in \mathbb{R}$.

Hence $\sum_{i=-\infty}^{\infty} \Psi(i+x)=1, \quad \forall x \in \mathbb{R}$, and $\sum_{i=-\infty}^{\infty} \Psi(x+i)=1, \quad \forall x \in \mathbb{R}$.

Theorem 11. It holds $\int_{-\infty}^{\infty} \Psi(x) d x=1$.

So $\Psi(x)$ is a density function on $\mathbb{R}$.

Theorem 12. Let $0<\beta<1$ and $n \in \mathbb{N}$. It holds

$$
\left\{\begin{array}{c}
\sum_{k=-\infty}^{\infty} \Psi(n x-k) \leq e^{4} \cdot e^{-2 n^{(1-\beta)}} . \\
:|n x-k| \geq n^{1-\beta}
\end{array}\right.
$$

Theorem 13. Let $x \in[a, b] \subset \mathbb{R}$ and $n \in \mathbb{N}$ so that $\lceil n a\rceil \leq\lfloor n b\rfloor$. It holds

$$
\frac{1}{\sum_{k=\lceil n a\rceil}^{\lfloor n b\rfloor} \Psi(n x-k)}<4.1488766=\frac{1}{\Psi(1)} .
$$

Also by [8, we obtain

$$
\lim _{n \rightarrow \infty} \sum_{k=\lceil n a\rceil}^{\lfloor n b\rfloor} \Psi(n x-k) \neq 1
$$

for at least some $x \in[a, b]$.

Definition 14. Let $f \in C([a, b])$ and $n \in \mathbb{N}$ such that $\lceil n a\rceil \leq\lfloor n b\rfloor$.

We further study, as in [8], the quasi-interpolation positive linear neural network operator

$$
F_{n}(f, x):=\frac{\sum_{k=\lceil n a\rceil}^{\lfloor n b\rfloor} f\left(\frac{k}{n}\right) \Psi(n x-k)}{\sum_{k=\lceil n a\rceil}^{\lfloor n b\rfloor} \Psi(n x-k)}, \quad x \in[a, b] .
$$

We find here fractional Voronovskaya type asymptotic expansions for $G_{n}(f, x)$ and $F_{n}(f, x)$, $x \in[a, b]$.

For related work on neural networks also see [1], [6], 9] and [10]. For neural networks in general see [16], [17] and [18]. 


\section{Main Results}

We present our first main result

Theorem 15. Let $\alpha>0, N \in \mathbb{N}, N=\lceil\alpha\rceil, f \in A C^{N}([a, b]), 0<\beta<1, x \in[a, b], n \in \mathbb{N}$ large enough. Assume that $\left\|D_{x-}^{\alpha} f\right\|_{\infty,[a, x]},\left\|D_{* x}^{\alpha} f\right\|_{\infty,[x, b]} \leq M, M>0$. Then

$$
G_{n}(f, x)-f(x)=\sum_{j=1}^{N-1} \frac{f^{(j)}(x)}{j !} G_{n}\left((\cdot-x)^{j}\right)(x)+o\left(\frac{1}{n^{\beta(\alpha-\varepsilon)}}\right),
$$

where $0<\varepsilon \leq \alpha$.

If $\mathrm{N}=1$, the sum in (17) collapses.

The last (17) implies that

$$
n^{\beta(\alpha-\varepsilon)}\left[G_{n}(f, x)-f(x)-\sum_{j=1}^{N-1} \frac{f^{(j)}(x)}{j !} G_{n}\left((\cdot-x)^{j}\right)(x)\right] \rightarrow 0,
$$

as $\mathrm{n} \rightarrow \infty, 0<\varepsilon \leq \alpha$.

When $N=1$, or $f^{(j)}(x)=0, j=1, \ldots, N-1$, then we derive that

$$
n^{\beta(\alpha-\varepsilon)}\left[G_{n}(f, x)-f(x)\right] \rightarrow 0
$$

as $n \rightarrow \infty, 0<\varepsilon \leq \alpha$. Of great interest is the case of $\alpha=\frac{1}{2}$.

Proof. From [13], p. 54; (5), we get by the left Caputo fractional Taylor formula that

$$
f\left(\frac{k}{n}\right)=\sum_{j=0}^{N-1} \frac{f^{(j)}(x)}{j !}\left(\frac{k}{n}-x\right)^{j}+\frac{1}{\Gamma(\alpha)} \int_{x}^{\frac{k}{n}}\left(\frac{k}{n}-J\right)^{\alpha-1} D_{* x}^{\alpha} f(J) d J,
$$

for all $x \leq \frac{k}{n} \leq b$.

Also from [3]; (6), using the right Caputo fractional Taylor formula we get

$$
f\left(\frac{k}{n}\right)=\sum_{j=0}^{N-1} \frac{f^{(j)}(x)}{j !}\left(\frac{k}{n}-x\right)^{j}+\frac{1}{\Gamma(\alpha)} \int_{\frac{k}{n}}^{x}\left(J-\frac{k}{n}\right)^{\alpha-1} D_{x-}^{\alpha} f(J) d J,
$$

for all $a \leq \frac{k}{n} \leq x$.

We call

$$
V(x):=\sum_{k=\lceil n a\rceil}^{\lfloor n b\rfloor} \Phi(n x-k)
$$

Hence we have

$$
\frac{f\left(\frac{k}{n}\right) \Phi(n x-k)}{V(x)}=\sum_{j=0}^{N-1} \frac{f^{(j)}(x)}{j !} \frac{\Phi(n x-k)}{V(x)}\left(\frac{k}{n}-x\right)^{j}+
$$




$$
\frac{\Phi(n x-k)}{V(x) \Gamma(\alpha)} \int_{x}^{\frac{k}{n}}\left(\frac{k}{n}-J\right)^{\alpha-1} D_{* x}^{\alpha} f(J) d J
$$

all $x \leq \frac{k}{n} \leq b$, iff $\lceil n x\rceil \leq k \leq\lfloor n b\rfloor$, and

$$
\begin{gathered}
\frac{f\left(\frac{k}{n}\right) \Phi(n x-k)}{V(x)}=\sum_{j=0}^{N-1} \frac{f^{(j)}(x)}{j !} \frac{\Phi(n x-k)}{V(x)}\left(\frac{k}{n}-x\right)^{j}+ \\
\frac{\Phi(n x-k)}{V(x) \Gamma(\alpha)} \int_{\frac{k}{n}}^{x}\left(J-\frac{k}{n}\right)^{\alpha-1} D_{x-}^{\alpha} f(J) d J,
\end{gathered}
$$

for all $a \leq \frac{k}{n} \leq x$, iff $\lceil n a\rceil \leq k \leq\lfloor n x\rfloor$.

We have that $\lceil n x\rceil \leq\lfloor n x\rfloor+1$.

Therefore it holds

$$
\begin{aligned}
& \sum_{k=\lfloor n x\rfloor+1}^{\lfloor n b\rfloor} \frac{f\left(\frac{k}{n}\right) \Phi(n x-k)}{V(x)}=\sum_{j=0}^{N-1} \frac{f^{(j)}(x)}{j !} \sum_{k=\lfloor n x\rfloor+1}^{\lfloor n b\rfloor} \frac{\Phi(n x-k)\left(\frac{k}{n}-x\right)^{j}}{V(x)}+ \\
& \quad \frac{1}{\Gamma(\alpha)}\left(\frac{\sum_{k=\lfloor n x\rfloor+1}^{\lfloor n b\rfloor} \Phi(n x-k)}{V(x)} \int_{x}^{\frac{k}{n}}\left(\frac{k}{n}-J\right)^{\alpha-1} D_{* x}^{\alpha} f(J) d J\right),
\end{aligned}
$$

and

$$
\begin{gathered}
\sum_{k=\lceil n a\rceil}^{\lfloor n x\rfloor} f\left(\frac{k}{n}\right) \frac{\Phi(n x-k)}{V(x)}=\sum_{j=0}^{N-1} \frac{f^{(j)}(x)}{j !} \sum_{k=\lceil n a\rceil}^{\lfloor n x\rfloor} \frac{\Phi(n x-k)}{V(x)}\left(\frac{k}{n}-x\right)^{j}+ \\
\frac{1}{\Gamma(\alpha)}\left(\sum_{k=\lceil n a\rceil}^{\lfloor n x\rfloor} \frac{\Phi(n x-k)}{V(x)} \int_{\frac{k}{n}}^{x}\left(J-\frac{k}{n}\right)^{\alpha-1} D_{x-}^{\alpha} f(J) d J\right) .
\end{gathered}
$$

Adding the last two equalities (24) and (25) we obtain

$$
\begin{gathered}
G_{n}(f, x)=\sum_{k=\lceil n a\rceil}^{\lfloor n b\rfloor} f\left(\frac{k}{n}\right) \frac{\Phi(n x-k)}{V(x)}= \\
\sum_{j=0}^{N-1} \frac{f^{(j)}(x)}{j !} \sum_{k=\lceil n a\rceil}^{\lfloor n b\rfloor} \frac{\Phi(n x-k)}{V(x)}\left(\frac{k}{n}-x\right)^{j}+ \\
\Gamma(\alpha) V(x) \\
\sum_{k=\lceil n a\rceil}^{\lfloor n x\rfloor} \Phi(n x-k) \int_{\frac{k}{n}}^{x}\left(J-\frac{k}{n}\right)^{\alpha-1} D_{x-}^{\alpha} f(J) d J+ \\
\left.\sum_{k=\lfloor n x\rfloor+1}^{\lfloor n b\rfloor} \Phi(n x-k) \int_{x}^{\frac{k}{n}}\left(\frac{k}{n}-J\right)^{\alpha-1}\left(D_{* x}^{\alpha} f(J)\right) d J\right\} .
\end{gathered}
$$


So we have derived

$$
T(x):=G_{n}(f, x)-f(x)-\sum_{j=1}^{N-1} \frac{f^{(j)}(x)}{j !} G_{n}\left((\cdot-x)^{j}\right)(x)=\theta_{n}^{*}(x),
$$

where

$$
\begin{aligned}
\theta_{n}^{*}(x): & =\frac{1}{\Gamma(\alpha) V(x)}\left\{\sum_{k=\lceil n a\rceil}^{\lfloor n x\rfloor} \Phi(n x-k) \int_{\frac{k}{n}}^{x}\left(J-\frac{k}{n}\right)^{\alpha-1} D_{x-}^{\alpha} f(J) d J\right. \\
& \left.+\sum_{k=\lfloor n x\rfloor+1}^{\lfloor n b\rfloor} \Phi(n x-k) \int_{x}^{\frac{k}{n}}\left(\frac{k}{n}-J\right)^{\alpha-1} D_{* x}^{\alpha} f(J) d J\right\} .
\end{aligned}
$$

We set

$$
\theta_{1 n}^{*}(x):=\frac{1}{\Gamma(\alpha)}\left(\frac{\sum_{k=\lceil n a\rceil}^{\lfloor n x\rfloor} \Phi(n x-k)}{V(x)} \int_{\frac{k}{n}}^{x}\left(J-\frac{k}{n}\right)^{\alpha-1} D_{x-}^{\alpha} f(J) d J\right),
$$

and

$$
\theta_{2 n}^{*}:=\frac{1}{\Gamma(\alpha)}\left(\frac{\sum_{k=\lfloor n x\rfloor+1}^{\lfloor n b\rfloor} \Phi(n x-k)}{V(x)} \int_{x}^{\frac{k}{n}}\left(\frac{k}{n}-J\right)^{\alpha-1} D_{* x}^{\alpha} f(J) d J\right)
$$

i.e.

$$
\theta_{n}^{*}(x)=\theta_{1 n}^{*}(x)+\theta_{2 n}^{*}(x) .
$$

We assume $b-a>\frac{1}{n^{\beta}}, 0<\beta<1$, which is always the case for large enough $n \in \mathbb{N}$, that is when $n>\left\lceil(b-a)^{-\frac{1}{\beta}}\right\rceil$. It is always true that either $\left|\frac{k}{n}-x\right| \leq \frac{1}{n^{\beta}}$ or $\left|\frac{k}{n}-x\right|>\frac{1}{n^{\beta}}$.

For $k=\lceil n a\rceil, \ldots,\lfloor n x\rfloor$, we consider

$$
\begin{gathered}
\gamma_{1 k}:=\left|\int_{\frac{k}{n}}^{x}\left(J-\frac{k}{n}\right)^{\alpha-1} D_{x-}^{\alpha} f(J) d J\right| \leq \\
\int_{\frac{k}{n}}^{x}\left(J-\frac{k}{n}\right)^{\alpha-1}\left|D_{x-}^{\alpha} f(J)\right| d J \\
\leq\left\|D_{x-}^{\alpha} f\right\|_{\infty,[a, x]} \frac{\left(x-\frac{k}{n}\right)^{\alpha}}{\alpha} \leq\left\|D_{x-}^{\alpha} f\right\|_{\infty,[a, x]} \frac{(x-a)^{\alpha}}{\alpha} .
\end{gathered}
$$

That is

$$
\gamma_{1 k} \leq\left\|D_{x-}^{\alpha} f\right\|_{\infty,[a, x]} \frac{(x-a)^{\alpha}}{\alpha}
$$

for $k=\lceil n a\rceil, \ldots,\lfloor n x\rfloor$.

Also we have in case of $\left|\frac{k}{n}-x\right| \leq \frac{1}{n^{\beta}}$ that

$$
\gamma_{1 k} \leq \int_{\frac{k}{n}}^{x}\left(J-\frac{k}{n}\right)^{\alpha-1}\left|D_{x-}^{\alpha} f(J)\right| d J
$$




$$
\leq\left\|D_{x-}^{\alpha} f\right\|_{\infty,[a, x]} \frac{\left(x-\frac{k}{n}\right)^{\alpha}}{\alpha} \leq\left\|D_{x-}^{\alpha} f\right\|_{\infty,[a, x]} \frac{1}{n^{\alpha \beta} \alpha} .
$$

So that, when $\left(x-\frac{k}{n}\right) \leq \frac{1}{n^{\beta}}$, we get

$$
\gamma_{1 k} \leq\left\|D_{x-}^{\alpha} f\right\|_{\infty,[a, x]} \frac{1}{\alpha n^{a \beta}} .
$$

Therefore

$$
\begin{aligned}
& \left|\theta_{1 n}^{*}(x)\right| \leq \frac{1}{\Gamma(\alpha)}\left(\frac{\sum_{k=\lceil n a\rceil}^{\lfloor n x\rfloor} \Phi(n x-k)}{V(x)} \gamma_{1 k}\right)=\frac{1}{\Gamma(\alpha)}
\end{aligned}
$$

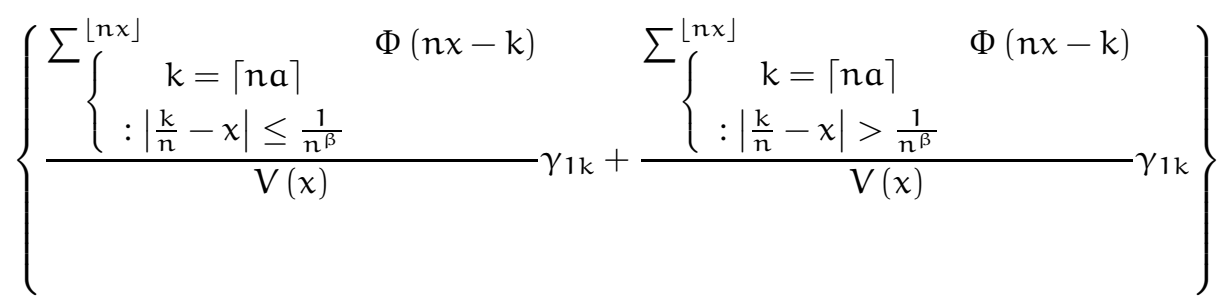

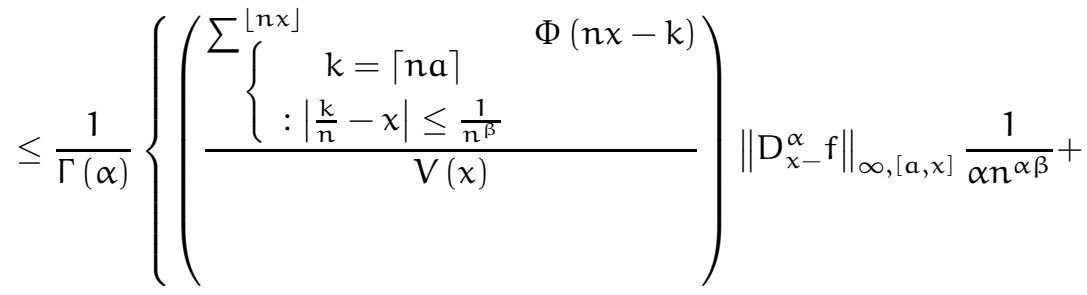

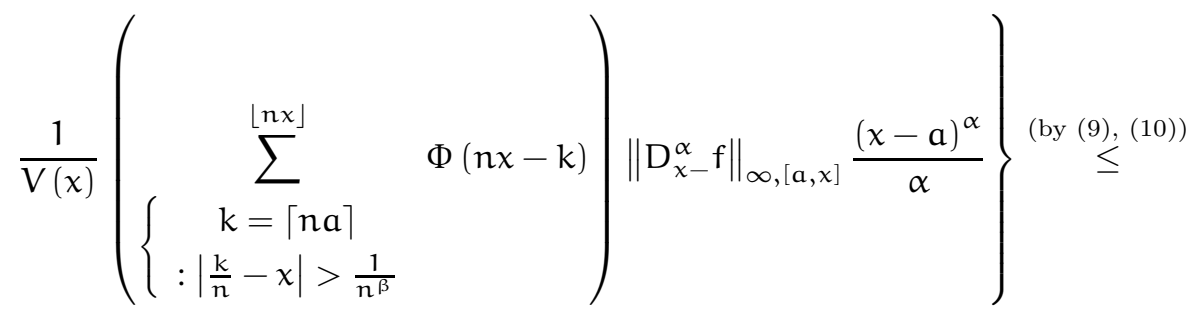

$$
\begin{aligned}
& \frac{\left\|D_{x-}^{\alpha} f\right\|_{\infty,[a, x]}}{\Gamma(\alpha+1)}\left\{\frac{1}{n^{\alpha \beta}}+(5.250312578)(3.1992) e^{-n^{(1-\beta)}}(x-a)^{\alpha}\right\} .
\end{aligned}
$$

Therefore we proved

$$
\left|\theta_{1 n}^{*}(x)\right| \leq \frac{\left\|D_{x-}^{\alpha} f\right\|_{\infty,[a, x]}}{\Gamma(\alpha+1)}\left\{\frac{1}{n^{\alpha \beta}}+(16.7968) e^{-n^{(1-\beta)}}(x-a)^{\alpha}\right\} .
$$

But for large enough $n \in \mathbb{N}$ we get

$$
\left|\theta_{1 n}^{*}(x)\right| \leq \frac{2\left\|D_{x-}^{\alpha} f\right\|_{\infty,[a, x]}}{\Gamma(\alpha+1) n^{\alpha \beta}} .
$$


Similarly we have

$$
\begin{gathered}
\gamma_{2 k}:=\left|\int_{x}^{\frac{k}{n}}\left(\frac{k}{n}-J\right)^{\alpha-1} D_{* x}^{\alpha} f(J) d J\right| \leq \\
\int_{x}^{\frac{k}{n}}\left(\frac{k}{n}-J\right)^{\alpha-1}\left|D_{* x}^{\alpha} f(J)\right| d J \leq \\
\left\|D_{* x}^{\alpha} f\right\|_{\infty,[x, b]} \frac{\left(\frac{k}{n}-x\right)^{\alpha}}{\alpha} \leq\left\|D_{* x}^{\alpha} f\right\|_{\infty,[x, b]} \frac{(b-x)^{\alpha}}{\alpha} .
\end{gathered}
$$

That is

$$
\gamma_{2 k} \leq\left\|D_{* x}^{\alpha} f\right\|_{\infty,[x, b]} \frac{(b-x)^{\alpha}}{\alpha}
$$

for $k=\lfloor n x\rfloor+1, \ldots,\lfloor n b\rfloor$.

Also we have in case of $\left|\frac{k}{n}-x\right| \leq \frac{1}{n^{\beta}}$ that

$$
\gamma_{2 k} \leq \frac{\left\|D_{* x}^{\alpha} f\right\|_{\infty,[x, b]}}{\alpha n^{\alpha \beta}}
$$

Consequently it holds

$$
\begin{aligned}
& \left|\theta_{2 n}^{*}(x)\right| \leq \frac{1}{\Gamma(\alpha)}\left(\frac{\sum_{k=\lfloor n x\rfloor+1}^{\lfloor n b\rfloor} \Phi(n x-k)}{V(x)} \gamma_{2 k}\right)= \\
& \frac{1}{\Gamma(\alpha)}\left\{\left(\begin{array}{c}
\sum^{\lfloor n b\rfloor} \begin{array}{c}
k=\lfloor n x\rfloor+1 \\
:\left|\frac{k}{n}-x\right| \leq \frac{1}{n^{\beta}}
\end{array} \\
V(x)
\end{array}\right) \frac{\left\|D_{* x}^{\alpha} f\right\|_{\infty,[x, b]}}{\alpha n^{\alpha \beta}}+\right. \\
& \frac{1}{V(x)}\left(\sum_{\begin{array}{c}
k=\lfloor n x\rfloor+1 \\
:\left|\frac{k}{n}-x\right|>\frac{1}{n^{\beta}}
\end{array}(n x-k)}^{\left\lfloor n b D_{* x}^{\alpha} f \|_{\infty,[x, b]} \frac{(b-x)^{\alpha}}{\alpha}\right.}\right\} \leq \\
& \frac{\left\|D_{* x}^{\alpha} f\right\|_{\infty,[x, b]}}{\Gamma(\alpha+1)}\left\{\frac{1}{n^{\alpha \beta}}+(16.7968) e^{-n^{(1-\beta)}}(b-x)^{\alpha}\right\} \text {. }
\end{aligned}
$$

That is

$$
\left|\theta_{2 n}^{*}(x)\right| \leq \frac{\left\|D_{* x}^{\alpha} f\right\|_{\infty,[x, b]}}{\Gamma(\alpha+1)}\left\{\frac{1}{n^{\alpha \beta}}+(16.7968) e^{-n^{(1-\beta)}}(b-x)^{\alpha}\right\} .
$$

But for large enough $n \in \mathbb{N}$ we get

$$
\left|\theta_{2 n}^{*}(x)\right| \leq \frac{2\left\|D_{* x}^{\alpha} f\right\|_{\infty,[x, b]}}{\Gamma(\alpha+1) n^{\alpha \beta}}
$$


Since $\left\|D_{x-}^{\alpha} f\right\|_{\infty,[a, x]},\left\|D_{* x}^{\alpha} f\right\|_{\infty,[x, b]} \leq M, M>0$, we derive

$$
\left|\theta_{n}^{*}(x)\right| \leq\left|\theta_{1 n}^{*}(x)\right|+\left|\theta_{2 n}^{*}(x)\right| \stackrel{(\text { by }}{\stackrel{399}{\leq}, 45)} \frac{4 M}{\Gamma(\alpha+1) n^{\alpha \beta}} .
$$

That is for large enough $n \in \mathbb{N}$ we get

$$
|T(x)|=\left|\theta_{n}^{*}(x)\right| \leq\left(\frac{4 M}{\Gamma(\alpha+1)}\right)\left(\frac{1}{n^{\alpha \beta}}\right),
$$

resulting to

$$
|\mathrm{T}(\mathrm{x})|=\mathrm{O}\left(\frac{1}{\mathrm{n}^{\alpha \beta}}\right)
$$

and

$$
|\mathrm{T}(\mathrm{x})|=\mathrm{o}(1) .
$$

And, letting $0<\varepsilon \leq \alpha$, we derive

$$
\frac{|T(x)|}{\left(\frac{1}{n^{\beta(\alpha-\varepsilon)}}\right)} \leq\left(\frac{4 M}{\Gamma(\alpha+1)}\right)\left(\frac{1}{n^{\beta \varepsilon}}\right) \rightarrow 0,
$$

as $\mathrm{n} \rightarrow \infty$.

I.e.

proving the claim.

$$
|\mathrm{T}(x)|=o\left(\frac{1}{n^{\beta(\alpha-\varepsilon)}}\right)
$$

We present our second main result

Theorem 16. Let $\alpha>0, N \in \mathbb{N}, N=\lceil\alpha\rceil, f \in A C^{N}([a, b]), 0<\beta<1, x \in[a, b], n \in \mathbb{N}$ large enough. Assume that $\left\|D_{x-}^{\alpha} f\right\|_{\infty,[a, x]},\left\|D_{* x}^{\alpha} f\right\|_{\infty,[x, b]} \leq M, M>0$. Then

$$
F_{n}(f, x)-f(x)=\sum_{j=1}^{N-1} \frac{f^{(j)}(x)}{j !} F_{n}\left((\cdot-x)^{j}\right)(x)+o\left(\frac{1}{n^{\beta(\alpha-\varepsilon)}}\right),
$$

where $0<\varepsilon \leq \alpha$.

If $\mathrm{N}=1$, the sum in (52) collapses.

The last (52) implies that

$$
n^{\beta(\alpha-\varepsilon)}\left[F_{n}(f, x)-f(x)-\sum_{j=1}^{N-1} \frac{f^{(j)}(x)}{j !} F_{n}\left((\cdot-x)^{j}\right)(x)\right] \rightarrow 0,
$$

as $\mathrm{n} \rightarrow \infty, 0<\varepsilon \leq \alpha$.

When $N=1$, or $f^{(j)}(x)=0, j=1, \ldots, N-1$, then we derive that

$$
n^{\beta(\alpha-\varepsilon)}\left[F_{n}(f, x)-f(x)\right] \rightarrow 0
$$

as $n \rightarrow \infty, 0<\varepsilon \leq \alpha$. Of great interest is the case of $\alpha=\frac{1}{2}$. 
Proof. Similar to Theorem [15, using (13) and (14).

Received: December 2011. Revised: May 2012.

\section{References}

[1] G.A. Anastassiou, Rate of convergence of some neural network operators to the unit-univariate case, J. Math. Anal. Appli. 212 (1997), 237-262.

[2] G.A. Anastassiou, Quantitative Approximations, Chapman\&Hall/CRC, Boca Raton, New York, 2001.

[3] G.A. Anastassiou, On Right Fractional Calculus, Chaos, solitons and fractals, 42 (2009), 365376.

[4] G.A. Anastassiou, Fractional Differentiation Inequalities, Springer, New York, 2009.

[5] G. Anastassiou, Fractional Korovkin theory, Chaos, Solitons \& Fractals, Vol. 42, No. 4 (2009), 2080-2094.

[6] G.A. Anastassiou, Inteligent Systems: Approximation by Artificial Neural Networks, Intelligent Systems Reference Library, Vol. 19, Springer, Heidelberg, 2011.

[7] G.A. Anastassiou, Fractional representation formulae and right fractional inequalities, Mathematical and Computer Modelling, Vol. 54, no. 11-12 (2011), 3098-3115.

[8] G.A. Anastassiou, Univariate hyperbolic tangent neural network approximation, Mathematics and Computer Modelling, 53(2011), 1111-1132.

[9] G.A. Anastassiou, Multivariate hyperbolic tangent neural network approximation, Computers and Mathematics 61(2011), 809-821.

[10] G.A. Anastassiou, Multivariate sigmoidal neural network approximation, Neural Networks $24(2011), 378-386$.

[11] G.A. Anastassiou, Univariate sigmoidal neural network approximation, submitted for publication, accepted, J. of Computational Analysis and Applications, 2011.

[12] Z. Chen and F. Cao, The approximation operators with sigmoidal functions, Computers and Mathematics with Applications, 58 (2009), 758-765.

[13] K. Diethelm, The Analysis of Fractional Differential Equations, Lecture Notes in Mathematics 2004, Springer-Verlag, Berlin, Heidelberg, 2010.

[14] A.M.A. El-Sayed and M. Gaber, On the finite Caputo and finite Riesz derivatives, Electronic Journal of Theoretical Physics, Vol. 3, No. 12 (2006), 81-95. 
[15] G.S. Frederico and D.F.M. Torres, Fractional Optimal Control in the sense of Caputo and the fractional Noether's theorem, International Mathematical Forum, Vol. 3, No. 10 (2008), 479-493.

[16] S. Haykin, Neural Networks: A Comprehensive Foundation (2 ed.), Prentice Hall, New York, 1998.

[17] W. McCulloch and W. Pitts, A logical calculus of the ideas immanent in nervous activity, Bulletin of Mathematical Biophysics, 7 (1943), 115-133.

[18] T.M. Mitchell, Machine Learning, WCB-McGraw-Hill, New York, 1997. 